Turbine $[\mathrm{J}]$. Journal of Turbomachinery, 2004, 126(10) : 578-586

[9] JENNY P, ABHARI R S, ROSA M G, et al. Unsteady Rotor Hub Passage Vortex Behavior in the Presence of Purge Flow in an Axial Low Pressure Turbine[R]. ASME 2012-GT-69256

[10] REGINA K, KALFAS A I, ABHARI R S. Experimental Investigation of Purge Flow Effects on a High Pressure Turbine Stage [J]. Journal of Turbomachinery, 2014, 137(4): 041006-8

[11] SCHADLER R, KALFAS A I, ABHARI R S. Modulation and Radial Migration for Turbine Hub Modes by the Rim Seal Purge Flow $[\mathrm{R}]$. ASME 2016-GT-56661

[12] BEHR T. Control of Rotor Tip Leakage and Secondary Flow by Casing Air Injection in Unshrouded Axial Turbines[D]. Dresden: Dresden University of Technology, 2007

[13] SCHUEPBACH P. Influence of Rim Seal Purge Flow on Performance of an Endwall Profiled Axial Turbine[D]. Swiss: Swiss Federal Institute of Technology, 2009

[14] 张晶辉, 马宏伟. 轮缘封严气体对浴轮转子性能影响的非定常数值研究 $[J]$. 推进技术, 2014, 35(4): 470-478

ZHANG Jinghui, MA Hongwei. Unsteady Numerical Investigation for Effects of Rim Sealing Flow on Performance of a Turbine Rotor [J]. Journal of Propulsion Technology, 2014, 35(4) : 470-478 (in chinese)

\title{
Numerical Investigation of Unsteady Interaction between Rim Seal Flow and Upstream Vane
}

\author{
YANG Fan, ZHOU Li, WANG Zhanxue \\ (School of Power and Energy, Northwestern Polytechnical University, Xi'an 710072, China)
}

\begin{abstract}
To investigate unsteady interaction mechanism between the rim seal flow in turbine stator-rotor cavity and main flow, detailed unsteady numerical simulations of the flow field and unsteady characteristic of the vane were conducted under different rim seal mass flow rate. The results show the blockage effect resulting from the egress flow leads to the pressure to increase and static entropy to reduce on the latter half of suction side near the hub. From the case without a cavity to $R_{I}=1.7 \%$, at $5 \%$ span, the maximum pressure coefficient increase on the suction side reaches $6 \%$. Moreover, the blockage effect causes the velocity to decrease at vane exit. Furthermore, the rim seal flow results in the decrease in lateral pressure gradient, causing the strength of hub passage vortex and hub trailing shedding vortex to reduce. Without rim seal flow, the ingress flow contributes to decreasing unsteady fluctuation from the hub to $10 \%$ span. When there is rim seal flow, unsteady fluctuation continues to reduce due to coupling effects of the egress flow from the ingress and the egress flow form the cavity inlet.
\end{abstract}

Keywords : cavity; vane; ingress; egress; unsteady 


\title{
复合材料层合板界面裂纹能量释放率解析方法研究
}

\author{
郑伟玲，郑龙席
}

(西北工业大学 动力与能源学院, 陕西 西安 710072)

\begin{abstract}
摘 要: 为了研究界面裂纹在复合材料中是否扩展, 使用 Timoshenko 梁理论和局部广义力得到了三 点弯曲模型中裂纹两端的解析能量释放率。研究结果和有限元结果进行对比验证, 发现解析解和有 限元结果相吻合。结果表明: 裂纹在载荷点一侧时, 左端和右端的能量释放率相等; 当穿过载荷点以 后, 裂纹左端的能量释放率继续上升后逐渐下降, 裂纹右端的能量释放率则下降后上升; 当裂纹关于 载荷点对称时, 两端能量释放率相等。
\end{abstract}

\section{关 键 词:脱层;能量释放率;裂纹;梁理论;复合材料层合板}

\section{中图分类号:0341}

文献标志码:A

复合材料具有高强度、高刚度、低密度和设计性 强的优点, 因此在航空工业得到广泛应用。然而, 由 于其在横向的层间强度较弱, 当有面外载荷的时候 在层与层界面上非常容易产生脱层。对复合材料层 合板中脱层扩展的研究大多采用断裂力学方法, 即 使用应变能量释放率 ( strain energy release rate, 简 称 SERR), 或者能量释放率 (简称 ERR) 作为所需 要确定的研究参数。

有限元方法 ${ }^{[1-5] 、 \text { 实验方法 }}{ }^{[6-9]}$ 及解析方法 ${ }^{[9-11]}$ 等用来分析复合材料层合板中裂纹的能量释放率 (ERR)。有限元方法或者实验方法的缺点在于每 次只能得到某一个具体模型的能量释放率, 这样相 对来说效率比较低, 增加了时间成本。由于有限元 方法和实验方法存在的缺点, 本文发展一种有效率 的解析方法。

多种描述复合材料层合板的理论可以用来计算 能量释放率。学者们 ${ }^{[12-15]}$ 使用梁理论来得到 I 型、 II 型或者混合型等裂纹的能量释放率, 其中 Timoshenko 梁理论能够得到较为精确的能量释放率。Wil$\operatorname{liams}^{[8]}$ 通过广义局部力来推导得到了层合板中脱 层的能量释放率。多数研究者 ${ }^{[6-9,12-13]}$ 的模型中裂 纹只是朝着一个方向扩展, 或者只是得到了不同类 型裂纹的能量释放率。在三点弯曲模型中, 裂纹随 2 个尖端何时扩展以及如何扩展都对结构有着显著
影响, 因此本文着重讨论如何解析求得三点弯曲模 型中裂纹 2 个尖端的能量释放率以及相关参数的 影响。

本文使用 Timoshenko 梁理论和 Williams ${ }^{[8]}$ 的方 法用来确定脱层的能量释放率, 能够快速高效的分 析复合材料层合板中脱层是否扩展以及扩展方向问 题。使用 Timoshenko 梁理论用来计算复合材料层 合板中脱层的能量释放率有可能降低精度但是可以 作为一种有效率的参数研究方法。

\section{1 模型简化}

复合材料层合板中有一个贯穿宽度方向的裂 纹,如图 1a) 所示, 基于切面可以简化为如图 1b) 所 示的二维问题。为得到复合材料层合板中裂纹的能 量释放率, 选择使用梁理论来描述在面外载荷作用 下的二维模型。在计算 SERR 的解析方法中, Williams ${ }^{[8]}$ SERR 使用裂纹尖端的广义力来计算双悬臂 梁中裂纹的 SERR 并得到精确的结果。对于三点弯 曲的模型来说, 图 $1 \mathrm{a}$ ) 是一般情况, 在厚度为 $t$, 宽度 为 $b$ 的复合材料层合板中有一个脱层或者说裂纹， 从上表面到裂纹的距离是 $t_{1}$ 。载荷在宽度方向简化 为均匀分布, 因此, 载荷在裂纹前端是均匀分布的。 
为了便于分析, 需要根据裂纹的数量以及裂纹是否 穿过载荷点把模型分为几个部分。例如裂纹没有穿 过载荷点,裂纹在载荷的左边, 如图 $1 \mathrm{~b})$ 所示, 整个 梁模型可以分为 5 个部分,其中 $0,3,4$ 是完整的梁, 1 和 2 是脱层后的次梁。

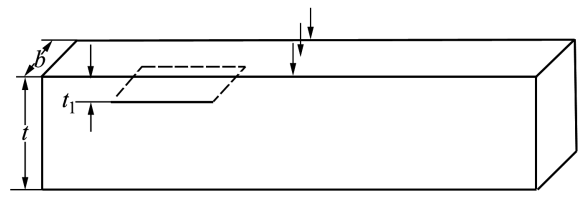

a）三维模型

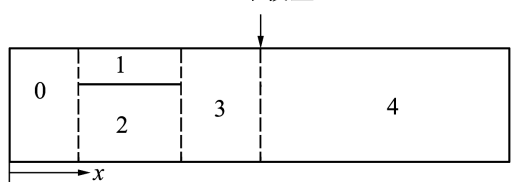

b）简化后的二维模型

图 1 裂纹未穿过载荷点

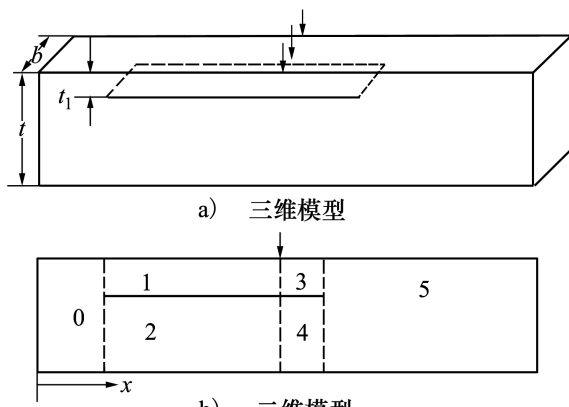

b) 二维模型

图 2 裂纹穿过载荷点

对于裂纹穿过载荷点的情况, 如图 2 所示, 则多 出来一个部分。对于分开的各个部分, 每个部分都 被当作单独的一个梁进行分析, 而且每一部分都必 须满足力和弯矩的平衡方程。

\section{2 推导过程}

本部分将以裂纹在载荷的一侧为例, 推导通过 位移方法求得三点弯曲模型中裂纹 2 个尖端的能量 释放率。Timoshenko 梁理论的控制方程是:

$$
\begin{gathered}
E I \frac{\mathrm{d}^{3} \phi}{\mathrm{d} x^{3}}=0 \\
\frac{\mathrm{d} w}{\mathrm{~d} x}=\phi-\frac{E I}{\kappa A G} \frac{\mathrm{d}^{2} \phi}{\mathrm{d} x^{2}}
\end{gathered}
$$

$\phi$ 是梁旋转的角度, $w$ 是位移, $E$ 是弹性模量, $I$ 是截
面惯性矩, $A$ 是截面面积, $G$ 是剪切模量, $\kappa$ 是和几何 形状有关的 Timoshenko 剪切系数, 当截面是矩形的 时候 $\kappa=\frac{5}{6}$ 。弯矩 $M$ 和剪力 $Q$ 的表达式为:

$$
\begin{gathered}
M=-D \frac{\mathrm{d} \phi}{\mathrm{d} x} \\
Q=S\left(\frac{\mathrm{d} w}{\mathrm{~d} x}-\phi\right)
\end{gathered}
$$

式中, $D$ 是梁的弯曲刚度, $D=E I$ 。 $S$ 则为剪切刚度, $S=\kappa A G_{\circ}$

对于没有裂纹的完整部分来说, 弯矩可以通过 方程 (3) 来计算得到。对于 0 和 3 部分的弯矩则可 以表示为

$$
M=-D \frac{\mathrm{d} \phi}{\mathrm{d} x}=\frac{P}{2} x
$$

方程(5)积分后可得

$$
\phi=-\frac{P x^{2}}{4 D}+C_{1}
$$

将(6)式代人方程 (2) 中积分一次可得

$$
w=-\frac{P x^{3}}{12 D}+\left(C_{1}+\frac{P}{2 S}\right) x+C_{2}
$$

编号为 4 的部分的 $\phi$ 和 $w$ 可以通过相同的流程得 到, 不同的是将弯矩的表达式改为 $M=P(L$ $-x) / 2$ 。

存在裂纹的次梁 1 和 2 的弯矩分布是未知的, 但是次梁 1 和 2 的转角表达式 $\phi$ 可以通过三次积分 方程 (1) 得到

$$
\phi=C_{0}+C_{1} x+C_{2} x^{2}
$$

将上面的转角表达式 $\phi$ 代人 (2) 式然后积分一次得 到位移表达式

$$
w=C_{3}+\left(C_{0}-2 \frac{D}{S} C_{2}\right) x+\frac{1}{2} C_{1} x^{2}+\frac{1}{3} C_{2} x^{3}
$$

如图 1b) 所示的 5 部分转角和位移表达式可以 写为:

$$
\begin{aligned}
& \phi_{0}=-\frac{P x^{2}}{4 D}+C_{01} \\
& w_{0}=-\frac{P x^{3}}{12 D}+\left(C_{01}+\frac{P}{2 S}\right) x+C_{02} \\
& \phi_{1}=C_{10}+C_{11} x+C_{12} x^{2} \\
& w_{1}=C_{13}+\left(C_{10}-2 \frac{D}{S} C_{12}\right) x+\frac{1}{2} C_{11} x^{2}+\frac{1}{3} C_{12} x^{3}
\end{aligned}
$$

$$
\phi_{2}=C_{20}+C_{21} x+C_{22} x^{2}
$$


$w_{2}=C_{23}+\left(C_{20}-2 \frac{D}{S} C_{22}\right) x+\frac{1}{2} C_{21} x^{2}+\frac{1}{3} C_{22} x^{3}$

$\phi_{3}=-\frac{P x^{2}}{4 D}+C_{31}$

$w_{3}=-\frac{P x^{3}}{12 D}+\left(C_{31}+\frac{P}{2 S}\right) x+C_{32}$

$\phi_{4}=\frac{P\left(x^{2}-2 x L\right)}{4 D}+C_{41}$

$w_{4}=\frac{P x^{3}}{12 D}-\frac{P L x^{2}}{4 D}+\left(C_{41}-\frac{P}{2 S}\right) x+C_{42}$

转角 $\phi$ 和位移 $w$ 的下标代表某一部分梁的编号。常 数 $C$ 的第一个下标为所表示梁的编号,第二个下标 则是为了便于区分。

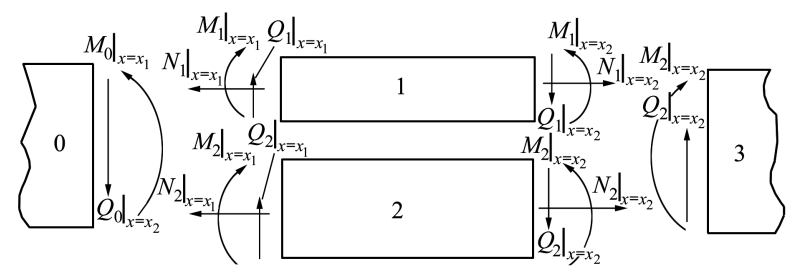

图 3 裂纹尖端的广义力

以裂纹没有穿过载荷点为例, 裂纹尖端部分的 广义内力分析如图 3 所示。 $\left.M_{1}\right|_{x=x_{1}},\left.Q_{1}\right|_{x=x_{1}}$ 和 $N_{1}$ $\mathrm{I}_{x=x_{1}}$ 是 1 梁在横截面 $x=x_{1}$ 处的弯矩、剪力和法向 力。下角标表示所在的部分, 坚线右边的部分表示 广义力所作用的未知。截面 $x=x_{1}$ 处的广义力连续 条件为:

$$
\begin{aligned}
& \left.N_{1}\right|_{x=x_{1}}+\left.N_{2}\right|_{x=x_{1}}=0 \\
& \left.Q_{1}\right|_{x=x_{1}}+\left.Q_{2}\right|_{x=x_{1}}-\left.Q_{0}\right|_{x=x_{1}}=0 \\
& \left.M_{1}\right|_{x=x_{1}}+\left.M_{2}\right|_{x=x_{1}}-\left.N_{1}\right|_{x=x_{1}} \frac{t_{1}}{2}+ \\
& \left.N_{2}\right|_{x=x_{1}} \frac{t_{2}}{2}=\left.M_{0}\right|_{x=x_{1}}
\end{aligned}
$$

式中, $t_{1}$ 和 $t_{2}$ 分别为次梁 1 和 2 的厚度, 且有 $t_{1}+t_{2}=$ $t$ 。在截面 $x=x_{2}$ 处的连续条件可以通过相似的方法 得到。次梁 1 在 $x$ 方向的平衡方程为

$$
\left.N_{1}\right|_{x=x_{1}}=\left.N_{1}\right|_{x=x_{2}}
$$

从(20) 式可以得出 $\left.N_{1}\right|_{x=x_{1}}=-\left.N_{2}\right|_{x=x_{1}}$, 联合 (23) 式可以得到:

$$
\begin{gathered}
\left.N_{1}\right|_{x=x_{1}}=\left.N_{1}\right|_{x=x_{2}}=N \\
\left.N_{2}\right|_{x=x_{1}}=\left.N_{2}\right|_{x=x_{2}}=-N
\end{gathered}
$$

式中, $N$ 是由于裂纹的存在而引起的法向力的值。
未损伤部分的梁的弯矩 $M$ 和剪力 $Q$ 的表达 式为:

$$
\begin{aligned}
& M_{0}(x)=\frac{P}{2} x \quad M_{3}(x)=\frac{P}{2} x \\
& M_{4}(x)=\frac{P}{2}(L-x) \\
& Q_{0}(x)=\frac{P}{2} \quad Q_{3}(x)=\frac{P}{2} \\
& Q_{4}(x)=-\frac{P}{2}
\end{aligned}
$$

将(24) 式 (26) 式代人弯矩连续方程 (22) 式以后 可以得到

$$
\left.M_{1}\right|_{x=x_{1}}+\left.M_{2}\right|_{x=x_{1}}-N \frac{t}{2}-\frac{P}{2} x_{1}=0
$$

两端的边界条件分别为

$$
\left.w_{0}\right|_{x=0}=\left.0 \quad w_{4}\right|_{x=L}=0
$$

在 $x=x_{1}$ 和 $x=x_{2}$ 处的位移和转角连续条件为:

$$
\begin{array}{ll}
\left.w_{0}\right|_{x=x_{1}}=\left.\left.w_{1}\right|_{x=x_{1}} \quad w_{0}\right|_{x=x_{1}}=\left.w_{2}\right|_{x=x_{1}} \\
\left.w_{3}\right|_{x=x_{2}}=\left.w_{1}\right|_{x=x_{2}} & \left.w_{3}\right|_{x=x_{2}}=\left.w_{2}\right|_{x=x_{2}} \\
\left.\phi_{0}\right|_{x=x_{1}}=\left.\phi_{1}\right|_{x=x_{1}} & \left.\phi_{0}\right|_{x=x_{1}}=\left.\phi_{2}\right|_{x=x_{1}} \\
\left.\phi_{3}\right|_{x=x_{2}}=\left.\phi_{1}\right|_{x=x_{2}} & \left.\phi_{3}\right|_{x=x_{2}}=\left.\phi_{2}\right|_{x=x_{2}}
\end{array}
$$

在 $x=\frac{L}{2}$ 处的位移和转角连续条件是:

$$
\begin{aligned}
& \left.w_{3}\right|_{x=\frac{L}{2}}=\left.w_{4}\right|_{x=\frac{L}{2}} \\
& \left.\phi_{3}\right|_{x=\frac{L}{2}}=\left.\phi_{4}\right|_{x=\frac{L}{2}}
\end{aligned}
$$

通过文献 $[1]$ 可以将变形后的梁的轴向平衡方 程可以写做

$$
\frac{N a}{E A_{2}}+\frac{N a}{E A_{1}}=\left(\left.\phi_{0}\right|_{x=x_{1}}-\left.\phi_{3}\right|_{x=x_{2}}\right) \frac{t_{1}+t_{2}}{2}
$$

将 (34) 式代人 (22) 式, 连续弯矩方程可以重新写为

$$
\begin{aligned}
& \left.M_{0}\right|_{x=x_{1}}=\left.M_{1}\right|_{x=x_{1}}+\left.M_{2}\right|_{x=x_{1}}+ \\
& \frac{t^{2}}{4 a}\left(\left.\phi_{0}\right|_{x=x_{1}}-\left.\phi_{3}\right|_{x=x_{2}}\right) \frac{E A_{1} E A_{2}}{E A_{1}+E A_{2}}
\end{aligned}
$$

如图 1b) 所示, 长度为 $a$ 的裂纹的能量释放率 是裂纹左端和右端的能量释放率的和。当需要判断 裂纹是否扩展, 朝着哪个方向扩展的时候就需要分 别裂纹左端和右端的能量释放率。裂纹左端的广义 力 $M_{1} \mathrm{I}_{x=x_{1}}, M_{2} \mathrm{I}_{x=x_{1}}, M_{0} \mathrm{I}_{x=x_{1}}, Q_{1} \mathrm{I}_{x=x_{1}}, Q_{2} \mathrm{I}_{x=x_{1}}$, $\left.Q_{0}\right|_{x=x_{1}}$ 和 $N$ 决定了裂纹左端的能量释放率 $G_{L}$, 对于 裂纹右端的能量释放率 $G_{R}$ 是一样的道理。裂纹左 端的能量释放率 $G_{L}$ 和右端的能量释放率 $G_{R}$ 的表达 式分别是: 


$$
\begin{gathered}
G_{L}(x)=\frac{1}{2}\left[\frac{\left(\left.M_{1}\right|_{x=x_{1}}\right)^{2}}{E_{1} I_{1}}+\frac{\left(\left.M_{2}\right|_{x=x_{1}}\right)^{2}}{E_{2} I_{2}}-\right. \\
\frac{\left(\left.M_{0}\right|_{x=x_{1}}\right)^{2}}{E_{0} I_{0}}+\frac{\left(\left.V_{1}\right|_{x=x_{1}}\right)^{2}}{\alpha_{s} G_{1} A_{1}}+\frac{\left(\left.V_{2}\right|_{x=x_{1}}\right)^{2}}{\alpha_{s} G_{2} A_{2}}- \\
\left.\frac{\left(\left.V_{0}\right|_{x=x_{1}}\right)^{2}}{\alpha_{s} G_{0} A_{0}}+\frac{N^{2}}{E_{1} A_{1}}+\frac{N^{2}}{E_{2} A_{2}}\right] \\
G_{R}(x)=\frac{1}{2}\left[\frac{\left(\left.M_{1}\right|_{x=x_{2}}\right)^{2}}{E_{1} I_{1}}+\frac{\left(\left.M_{2}\right|_{x=x_{2}}\right)^{2}}{E_{2} I_{2}}-\right. \\
\frac{\left(\left.M_{3}\right|_{x=x_{2}}\right)^{2}}{E_{3} I_{3}}+\frac{\left(\left.V_{1}\right|_{x=x_{2}}\right)^{2}}{\alpha_{s} G_{1} A_{1}}+\frac{\left(\left.V_{2}\right|_{x=x_{2}}\right)^{2}}{\alpha_{s} G_{2} A_{2}}- \\
\left.\frac{\left(\left.V_{3}\right|_{x=x_{2}}\right)^{2}}{\alpha_{s} G_{3} A_{3}}+\frac{N^{2}}{E_{1} A_{1}}+\frac{N^{2}}{E_{2} A_{2}}\right]
\end{gathered}
$$

\section{3 验证和结果分析}

能量释放率 $G$, 是应变能的微分, 可以在求得所 有的未知数以后通过计算得到。Williams ${ }^{[8]}$ 得到了 左端受到集中点载荷右端固定的双悬臂梁模型, 即 $I I$ 型裂纹的能量释放率的表达式 $G_{I I}=\frac{9}{4} \frac{P^{2} a^{2}}{b^{2} E h^{3}}$, 其 中 $P$ 是施加的载荷, $a$ 是裂纹的长度, $b$ 是梁的宽度, $E$ 为材料的弹性模量, $h$ 为厚度的一半。在有限元软 件 ABAQUS 中, 可以通过 $J$ 积分来得到裂纹尖端的 能量释放率。在有限元模型中, 施加的载荷为 $P=$ $50 \mathrm{~N}$, 梁的长度为 $L=39.825 \mathrm{~mm}$, 厚度 $t=4.32 \mathrm{~mm}$,

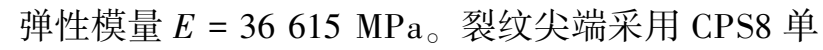
元, 尖端处网格大小为 $0.0067 \mathrm{~mm}$, 是梁厚度的 $0.155 \%$ 。有限元结果和 Williams 的结果进行对比 如图 4 所示, 非常吻合。

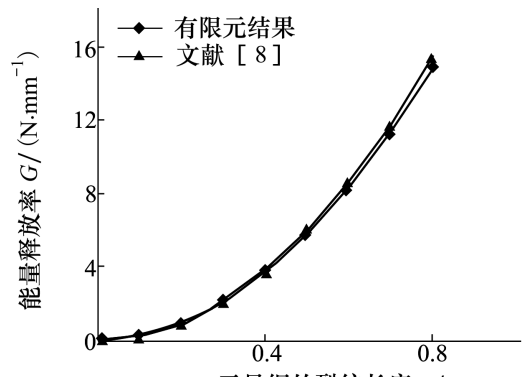

无量纲的裂纹长度 $a / L$

图 4 有限元结果和文献 $[8]$ 对比

裂纹在双悬臂梁模型里面只能朝着一个方向扩 展, 然而在三点弯曲模型, 如图 1 和图 2 所示, 则可
以朝着 2 个方向扩展。为了验证解析解, 使用有限 元软件创建了一个三点弯曲梁模型, 梁的长度为 $79.65 \mathrm{~mm}$, 厚度为 $1.44 \mathrm{~mm}$, 裂纹在厚度的 $1 / 3$ 位 置, 施加的载荷大小的 $1 \mathrm{~N}$, 材料为各项同性, 弹性 模量 $E=36615 \mathrm{MPa}$, 通过 $\mathrm{J}$ 积分来求得能量释放率 的有限元结果。通过使用本文提出的解析方法得到 的能量释放率同有限元得到的能量释放率的对比如 图 5 所示。 $x_{1}$ 表示裂纹左端的位置, $t_{1} / t=1 / 3$ 表明 裂纹所在厚度方向的 $1 / 3$ 位置。结果表明解析的能 量释放率和有限元方法得到的能量释放率基本 一致。

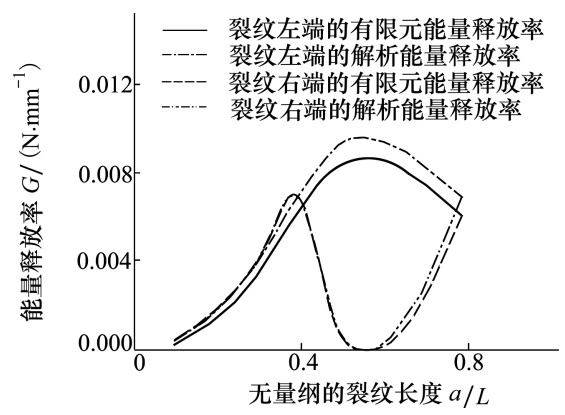

图 5 有限元结果和解析结果对比

裂纹两端的能量释放率如图 6 所示。从图 6 可 以看出, 当整个裂纹位于载荷的一侧时, 裂纹左端和 右端的能量释放率相等, 经过中间的载荷点以后, 左 端的能量释放率继续上升, 到达极值以后开始下降, 而右端的能量释放率则在经过载荷点后开始下降, 到达极小值后开始增大。当裂纹长度关于载荷点对 称时,左右两端的能量释放率再次相等。

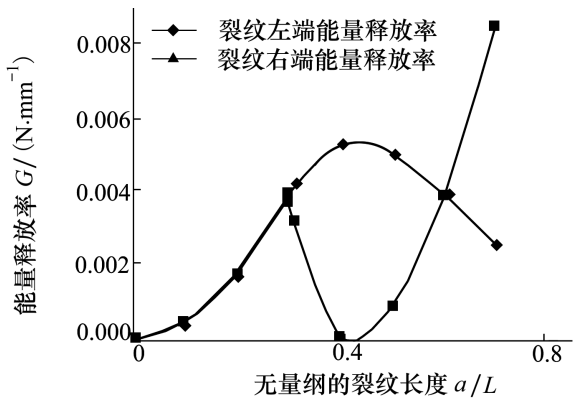

图 6 裂纹左右两端的能量释放率

裂纹左端位置对裂纹左右两端能量释放率的影 响如图 7 和图 8 所示。结果表明: 裂纹左端距离载 荷点越小, 左端的能量释放率越小; 对于裂纹右端的 能量释放率来说, 裂纹左端距离载荷点越小, 右端的 能量释放率在裂纹超过载荷点一定长度 (约 $0.1 L$ ) 
以后,增加的速度非常快。

当脱层出现在复合材料中, 则可以将脱层当作 裂纹,然后将复合材料层合板分为如图 2 所示的几 个部分, 求得等效刚度参数后即可通过本文提供的 方法求得脱层两端的能量释放率, 从而为判断脱层 是否扩展提供依据。为验证通过此方法得到的复合 材料层合板中裂纹的能量释放率, 建立了铺层顺序 为 $\left[90_{5} / 0_{5} / 90 / 0\right] \mathrm{s}$ 的 24 层层合板有限元模型。

模型中材料的性能参数和 AS4/8552 一致, $0^{\circ}$ 铺 层的材料属性为: $E_{1}=148 \mathrm{GPa}, E_{2}=E_{3}=9.65 \mathrm{GPa}$, $G_{23}=3.64 \mathrm{GPa}, G_{12}=G_{13}=4.55 \mathrm{GPa}, \nu_{12}=\nu_{13}=0.3, \nu_{23}$ $=0.45$ 。层合板梁的长度 $L=79.65 \mathrm{~mm}$, 厚度 $t=4.32$ $\mathrm{mm}$, 载荷在梁的中间位置。裂纹厚度方向的位置为 上半部分的 $0^{\circ}$ 铺层和 $90^{\circ}$ 铺层的界面上, 裂纹左端 在 $0.2 L$ 位置处, 裂纹右端的位置随着裂纹长度 $a$ 的 变化而不同。有限元结果和解析的能量释放率的对

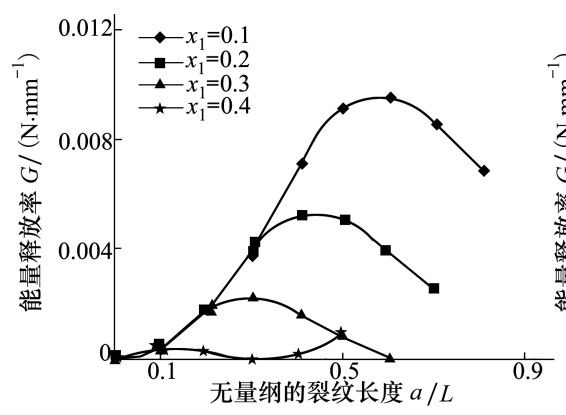

图 7 裂纹位置对裂纹左 端能量释放率的影响

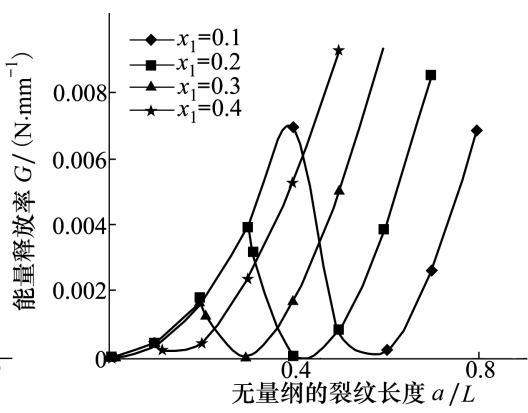

图 8 裂纹位置对裂纹右 端能量释放率的影响
比如图 9 所示。从图 9 可以看出, 通过解析方法得 到的复合材料层合板中裂纹左右两端的能量释放率 和有限元结果基本吻合, 且复合材料层合板中裂纹 两端能量释放率和各向同性材料中裂纹能量释放率 趋势变化一致。当裂纹长度 $a / L<0.25$ 时, 有限元结 果和解析结果差别约 $20 \%$ 。这种差别可能是由于 (1)Timoshenko 梁理论不适合小裂纹长度分析; (2)也 可能是小裂纹情况下, 厚度与长度比较大, 不能简化 为二维结构, 因此广义力模型分析的时候缺少对于 厚度方向的因素考虑; 3 在三点弯曲结构中, 认为 I 裂纹可以忽略不计, 主要考虑 II 型裂纹, 因此解析 模型中只包含 II 型裂纹分析。在有限元三维模型 中, $J$ 积分计算能量释放率时没有指定裂纹类型, 因 此小裂纹时差别较大可能受到了有限元计算中 I 型 裂纹能量释放率的影响。

\section{4 结 论}

本文发展了一种通过位移来计算得到三点弯曲 梁模型中的裂纹的能量释放率的方法, 对比解析结 果和有限元结果一致而得到验证。在实际应用中, 若材料的界面断裂韧性即临界能量释放率大于所分

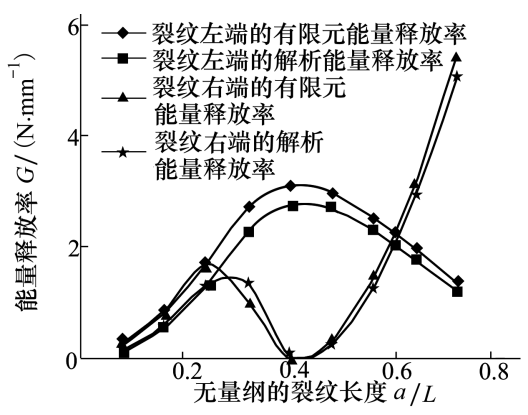

图 9 复合材料中裂纹中的能量释放率 的有限元结果和解析结果对比

\section{参考文献:}

[1] MASSABO R, COX B. Concepts for Bridged Mode II Delamination Cracks [J]. Journal of the Mechanics and Physics of Solids, $1999,47: 1265-1300$

[2] TUron A, COSTA J, CAmanho P, et al. Simulation of Delamination in Composites under High-Cycle Fatigue[J]. Composites Part A: Applied Science and Manufacturing, 2007, 38(11) : 2270-2282

[3] KRUEGER R. The Virtual Crack Closure Technique: History, Approach and Application[R]. NASA CR-2002-211628

[4] KRAUSE T, TUSHTEV K, KOCH D, GRATHWOHL G. Interlaminar Mode I Crack Growth Energy Release Rate of Carbon/ 
Carbon Composites [J]. Engineering Fracture Mechanics, 2013,100( 100) : 38-51

[5] ZHENG W, KASSAPOGLOU C. Prediction of Delamination Onset and Growth for Apply Composite Laminates Using the Finite Element Method $[\mathrm{J}]$. Composites Part A: Applied Science and Manufacturing, 2017, 101:381-393

[6] SAPONARA V, MULIANA H, HAJ-ALI R, et al. Experimental and Numerical Analysis of Delamination Growth in Double Cantilever Laminated Beams[J]. Engineering Fracture Mechanics, 2002, 69: 687-699

[7] CHEN I, PELEGRI A. Mixed Mode Testing of Fiber Reinforced Composites Using a Modified MMB Fixture[C]//41st Structures, Structaral Dynamics, and Materials Conference and Exhibit, 2000

[8] WILLIAMS J. On the Calculation of Energy Release Rate for Cracked Laminates [J]. International Journal of Fracture, 1988 , 36 (2) : 101-119

[9] SHEINMAN I, KARDOMATEAS G. Energy Release Rate and Stress Intensity Factors for Delaminated Composite Laminates [J]. International Journal of Solids and Structures, 1997,34(4):451-459

[10] OZDIL F, CARLSSON L. Beam analysis of Angle-Ply Laminate DCB Specimens[ J]. Composites Science and Technology, 1999, $59(2): 305-315$

[11] HAMED M, NOSIER A, FARRAHI G. Separation of Delamination Modes in Composite Beams with Symmetric Delaminations [J]. Materials \& Design, 2006,27: 900-910

[12] NAIRN J. On the calculation of Energy Release Rates for Cracked Laminates with Residual Stresses [J]. International Journal of Fracture, 2006,139:267-293

[13] ANDREWS M, MASSABO R, COX B. Elastic Interaction of Multiple Delaminations in Plates Subject to Cylindrical Bending[J]. International Journal of Solids and Structures, 2006,43(5):855-886

[14] SUN R, PANDEY R. Improved Method for Calculating Strain Energy Release Rate Based on Beam Theory[J]. AIAA Journal, $1994,32(1): 184-189$

[15] KASSAPOGLOU C. Design and Analysis of Composite Structures with Applications to Aerospace Structures[M]. UK: John Wiley \& Sons, 2010

\title{
Analytical Energy Release Rate of Interfacial Crack in Composite Laminates
}

\section{ZHENG Weiling, ZHENG Longxi}

(School of Power and Energy, Northwestern Polytechnical University, Xi'an 710072, China)

\begin{abstract}
In order to study whether the interfacial crack will grow or not in the composite laminates, the energy release rate of a crack in three-point bending model was obtained by using the Timoshenko beam theory and local generalized forces. The results of energy release rate were validated by the finite element results. The results indicate that the energy release rate of left crack tip is equal to that of the right crack tip when the crack before the crack goes cross the loading point; after the crack goes cross the loading point, the energy release rate of the left crack tip increases and then decreases gradually, while the energy release rate of right crack tip decreases first and increases later; the energy release rate of left crack tip is equal to that of the right crack tip again when the crack is symmetric with the loading point.
\end{abstract}

Keywords : delamination; energy release rate; crack; beam theory; composites laminate 


\title{
点云和视觉特征融合的增强现实装配系统 三维跟踪注册方法
}

\author{
王月，张树生，白晓亮
}

(西北工业大学 机电学院, 陕西 西安 710072)

\begin{abstract}
摘 要: 为了提高三维跟踪注册方法面向机械产品增强现实装配引导的适用性和鲁棒性, 提出了一种 点云和视觉特征融合的三维跟踪注册方法。首先利用参考模型点云对三维跟踪注册绝对坐标系进行 定义,从而确定虚拟装配引导信息的定位基准。然后在迭代最近点法点云数据配准基础上,结合深度 传感器彩色图像信息, 通过视觉特征匹配, 提高深度传感器快速移动时的跟踪注册过程鲁棒性。为了 在此过程获取足够数量的视觉特征匹配点对,提出了一种基于方向向量一致性的视觉特征匹配策略。 最后在跟踪注册过程加入基于关键帧的回环检测和全局位姿优化。实验结果表明: 新方法精确性、实 时性好, 能达到每秒 30 帧。而且在相机快速移动时仍能表现出较好的鲁棒性, 其综合性能优于基于 点云的 Kinect Fusion 方法。
\end{abstract}

\section{关 键 词:机械装配; 增强现实;三维跟踪注册;点云;视觉特征}

\section{中图分类号:TP391}

文献标志码:A

装配是产品生命周期中的一个重要环节,产品 的装配时间和装配质量直接影响着产品的开发成本 和使用性能。据统计, 在现代制造过程中, 装配工作 量占整个产品研制工作量的 $20 \% \sim 70 \%$, 平均为 $45 \%$,装配时间占整个制造时间的 $40 \% \sim 60 \%$ 。同 时, 产品装配通常以手工作业为主, 费用高昂且属于 产品研制工作的后端, 提高装配效率和可靠性所带 来的经济效益远比简单地降低零件生产成本所带来 的经济效益显著 ${ }^{[1]}$ 。

由于增强现实 $(\mathrm{AR})$ 技术具有同时展现真实世 界信息和虚拟世界信息的能力, 能够将计算机生成 的装配引导信息 (如三维模型、装配动画、文本提示 等) 在装配现场中 “无缝” 叠加, 能大大提高装配操 作效率和质量。因此, 近年来 $\mathrm{AR}$ 技术被越来越多 地应用到机械产品装配过程, 基于 AR 技术的装配 辅助系统层出不穷。其中, 精确的三维跟踪注册是 $\mathrm{AR}$ 装配辅助系统的核心技术之一, 它是实现虚拟 装配提示信息在装配环境中准确叠加的关键。经过 近几年的发展,三维跟踪注册已经取得了重大进步,
文章编号 : 1000-2758(2019) 01-0143-09
但仍然存在着不同程度的缺陷: 基于自然特征点的 方法 ${ }^{[2-3]}$ 在表面缺少足够纹理特征的环境中容易出 现跟踪注册抖动或扰动。基于边缘轮廓的方法 ${ }^{[4-5]}$ 对杂乱的背景比较敏感, 且对于装配交互过程中造 成的目标物体部分遮挡不鲁棒。基于模型的方 法 $^{[6-8]}$ 需要对从不同视角采集到的大量参考视图进 行处理,这会导致巨大的搜索空间和庞大的运算量, 大大降低了系统的实时性和可用性。基于即时定位 与地图构建 $(\text { SLAM })^{[9-11]}$ 方法的三维跟踪注册不需 要场景的先验知识, 免去了复杂的训练过程。但它 们仅能估计出相机的相对位姿, 对于需要绝对位置 信息的装配场景不能直接适用, 而且这些方法在动 态场景中容易造成跟踪注册失败。基于稠密点云的 三维跟踪注册方法 ${ }^{[12-13]}$ 能在光照强度较低且表面 缺少纹理的机械装配环境中表现出较好的鲁棒性。 该类方法运用 ICP (iterative closest point) 配准算法 对全部或部分点云进行迭代运算, 降低了对光照条 件的要求, 同时提高了传感器位姿估计精度。但是 这类方法与 SLAM 方法相同, 也仅能估计传感器的 
相对位姿。而且当深度传感器快速移动, 导致无法 获取正确的初始对应数据点集时, ICP 算法迭代过 程容易陷人局部最优, 难以保证收玫速度和配准精 度,甚至造成“丢帧” 的情况，使跟踪注册过程中断。 同时, 跟踪注册过程采用连续帧间配准会随着传感 器运动距离的增大, 跟踪注册精度大幅降低。

本文提出了一种精确、鲁棒的无标识三维跟踪 注册方法。首先以 ICP 点云配准为基础, 利用参考 模型点云对三维跟踪注册绝对坐标系进行定义。然 后结合彩色图像信息, 将视觉特征匹配点对作为 ICP 迭代过程的初始对应数据点集。为了在光滑、 无纹理的装配环境中获取足够数量的视觉特征匹配 点对,提出一种基于方向向量一致性的视觉特征匹 配策略,从而提高了装配环境中的跟踪注册算法精 确性和鲁棒性。

\section{1 系统概况}

算法基本思想和工作流程如图 1 所示。在离线 阶段,将参考 CAD 模型生成三维参考点云模型, 为 跟踪注册绝对坐标系的建立做准备。在在线阶段, 采集三维装配环境点云, 利用点云配准计算参考模 型点云和装配环境中物理对象表面点云之间的变换 关系, 完成绝对坐标系的定义。然后采用 RGBDICP 配准对跟踪注册过程相机位姿进行估计, 并利 用回环检测对估计位姿进行优化。最后根据三维跟 踪注册结果, 将装配引导信息在装配环境中叠加, 实 现装配过程的可视化引导。

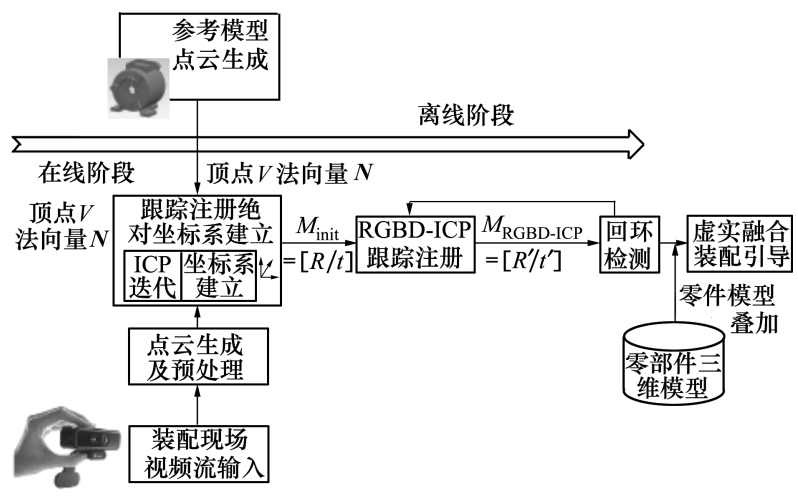

图 1 算法思想和工作流程

\section{1 点云生成}

首先采用 WJBF 方法 (weighted joint bilateral filter $)^{[14]}$ 对装配环境深度图像进行降噪修复。然后将
降噪后深度图像中 $\mathrm{i}$ 时刻的每一个像素点 $u=(x$, $y)$,利用 NVIDIA 公司的 CUDA 并行计算平台反投 影到深度相机空间坐标系, 获得顶点 (vertex map), 反投影结果为

$$
V_{i}(u)=D_{i}(u) M_{\text {int_D }}^{-1}[u, 1]
$$

式中, $D_{i}(u)$ 为 $i$ 时刻的深度图像, $M_{\mathrm{int}_{-} D}$ 为深度相机 内部参数。

在时刻 $i$ 的 6DOF 相机位姿用矩阵 $\boldsymbol{M}_{i}=\left[\boldsymbol{R}_{i} \mid \boldsymbol{t}_{i}\right]$ 表示, 其中 $\boldsymbol{R}_{i}$ 为 $3 \times 3$ 的旋转矩阵, $\boldsymbol{t}_{i}$ 为三维平移向 量。通过 6DOF 相机位姿, 可将 (1) 式转换到全局坐 标空间

$$
V_{3 \mathrm{D}}(u)=V_{i}(u) M_{i}
$$

同时, 每个顶点对应的法向量 $N_{3 \mathrm{D}}(u)$ 用相邻 投影点通过作如下运算得

$$
\begin{aligned}
& N_{3 \mathrm{D}}(u)=\left(V_{3 \mathrm{D}}(x+1, y)-V_{3 \mathrm{D}}(x, y)\right) \times \\
& \left(V_{3 \mathrm{D}}(x, y+1)-V_{3 \mathrm{D}}(x, y)\right)
\end{aligned}
$$

将(3) 归一化到单位长度 $N_{3 \mathrm{D}} /\left\|N_{3 \mathrm{D}}\right\|_{2}$ 。归一 化后的单位法向量 $N_{3 \mathrm{D}}(u)$ 和三维顶点 $V_{3 \mathrm{D}}(u)$ 构成 三维装配环境点云。

\section{2 三维跟踪注册绝对坐标系建立}

将参考对象三维模型在 CAD 环境中生成点云 $\boldsymbol{P}_{i}=\left\{p_{0}, p_{1}, p_{2} \cdots p_{m}, n_{0}, n_{1}, n_{2} \cdots n_{m}\right\}$, 其中 $p_{0}, p_{1}$, $\cdots p_{m}$ 为模型表面顶点坐标, $n_{0}, n_{1}, \cdots n_{m}$ 为顶点对应 的法向量。以水泵 AR 装配引导为例,将基座三维 模型作为参考对象, 生成点云, 获得表面顶点个数 5558 , 法向量数为 5078 (见图 2)。

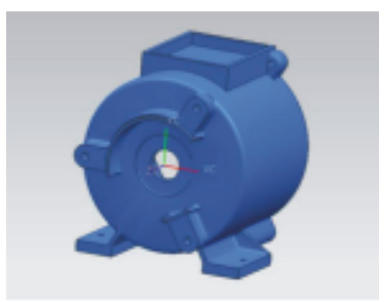

a）基座 $\mathrm{CAD}$ 模型

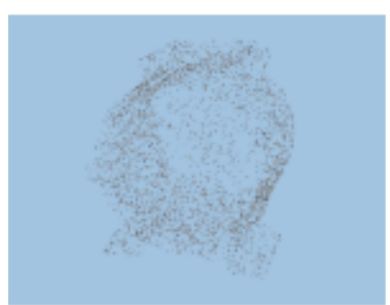

b）基座模型点云
图 2 基座模型点云生成

在线装配过程中, 首先将 $t$ 时刻降噪修复后的 装配现场深度图像 $R_{t}(u)$, 利用 1.1 节方法生成三维 环境点云 $\boldsymbol{X}$, 其中 $\boldsymbol{X}=\left\{x_{0}, x_{1}, x_{2} \cdots x_{n}, N_{0}, N_{1}\right.$, $\left.N_{2} \cdots N_{n}\right\}$ 。然后采用 ICP 算法, 将三维环境点云 $\boldsymbol{X}$ 与参考模型点云 $\boldsymbol{P}_{i}=\left\{p_{0}, p_{1}, p_{2} \cdots p_{m}, n_{0}, n_{1}, n_{2} \cdots n_{m}\right\}$ 进行配准。配准过程使用欧氏距离作为度量, 同时 引人法向量特征作为额外限制条件, 以尽量减少误 
匹配点数目。法向量之间的相似性用点积 $d_{i j}=$ $\left\langle N_{i}, n_{j}\right\rangle$ 进行表示, 假如 $d_{i j}$ 大于某一阈值 $\psi$, 则认为 是对应点, 并赋予权值 1 , 表示为

$$
w= \begin{cases}1, & d_{i j}>\psi \\ 0, & \text { 其他 }\end{cases}
$$

通过采用欧式距离和法向量特征度量方法, 便 可获得对应点的个数 $N$ 及每一对对应点的权值 $w_{i}$ 。采用最小二乘法对迭代过程中位置关系误差矩 阵进行求解, 其中误差关系矩阵为

$$
e_{i}(\boldsymbol{R}, \boldsymbol{t})=\frac{1}{N} \sum_{i=1}^{N_{p}} w_{i}\left\|x_{i}-R \cdot p_{i}-\boldsymbol{t}\right\|^{2}
$$

通过旋转矩阵 $\boldsymbol{R}$ 和平移向量 $\boldsymbol{t}$ 的求解, 便可获 得基座参考模型点云和真实基座点云重合时的变换 关系:

$$
\boldsymbol{M}_{\text {init }}=[\boldsymbol{R} \mid \boldsymbol{t}]
$$

假设参考对象模型坐标系原点即为相机坐标系 原点, 所以通过变换关系 $M_{\text {init }}$ 的求解即可获得相机 在参考模型坐标系 (即装配坐标系)下的初始位姿。 在上述计算过程中采用了大量点云数据参与计算, 虽然保证了始位姿矩阵的计算精度, 但同时大大增 加了时间消耗。因此, 本文仅采用此步骤进行相机 位姿的初始化, 初始化完成后的相机位姿将采用连 续视频帧间 ICP 点云配准的方法进行计算, 后文将 详细进行介绍。

\section{3 视觉特征提取与匹配}

连续视频帧间 ICP 点云配准是三维跟踪注册中 常用的方法。它主要分为 2 步: 第一步确定对应数

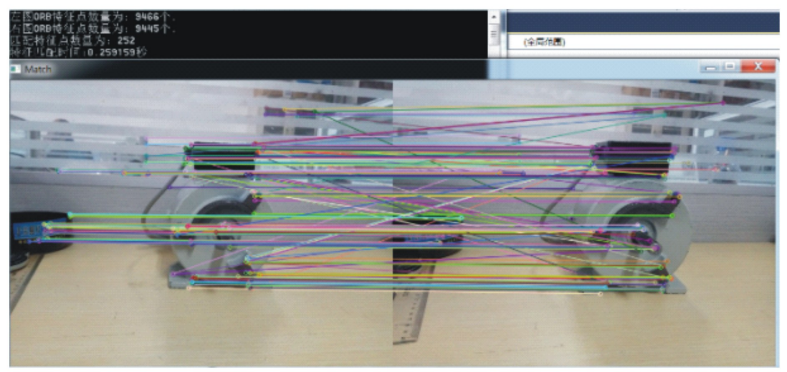

图 3 原始 ORB 特征提取与匹配

为了能获取足够数量的正确 ORB 特征点对作 为初始匹配数据点集, 本文受基于网格的运动统计 (GMS $)^{[15]}$ 方法思想启发一一运动平滑一致性的匹 配点不大可能是随机出现的, 匹配点对真假的判别 可以通过周围匹配点的简单计数来实现。基于上述 思想对 OBR 特征匹配过程进行改进, 本文称为
据点集,第二步根据对应点集求解点云数据间的变 换关系。但是现有方法在相机突然发生快速移动 时,ICP 配准算法由于无法获取前后 2 帧点云数据 间的初始对应关系,使计算出的相机位姿不准确,甚 至造成跟踪注册失败。考虑到 Softkinetic 彩色图像 数据和深度图像数据存在一一对应的关系, 可将点 云的初始匹配问题转化为彩色图像特征点的匹配问 题。利用匹配的视觉特征点对作为初始对应点集, 是解决连续视频帧间 ICP 点云配准的有效方法。

目前很多局部特征如 SIFT、SURF、ORB、 BRISK、FREAK 等被广泛应用于图像匹配、物体识 别等领域。基于特征点匹配的快速性和稳定性的考 虑,本文选用 ORB 局部特征。ORB 局部特征采用 FAST 作为特征点检测子, 改进的 BRIEF 作为特征 描述子,采用 $\mathrm{BF}$ (brute force) 模式匹配算法进行特 征描述符匹配,该算法对具有丰富纹理的物体图像 匹配表现出良好的性能。但是由于机械装配环境中 零部件表面光滑, 原始 ORB 匹配过程难以获得足够 数量的匹配点对, 且特征点比较集中, 不利于保证旋 转平移矩阵计算的可靠性。图 3 为采用原始 $\mathrm{ORB}$ 进行特征提取与匹配的结果。从图中可以看出, 原 始 ORB 匹配可以提取到足够数量的特征点, 但是匹 配点对较少, 且其中的误匹配较多, 常采用随机抽样 一致性检测 (RANSAC) 算法进行误匹配点剔除。图 4 为原始 $\mathrm{ORB}$ 特征匹配采用 RANSAC 后最终只能 获得 87 个匹配点对, 匹配点对数量进一步降低, 难 以满足 ICP 初始配准需求。

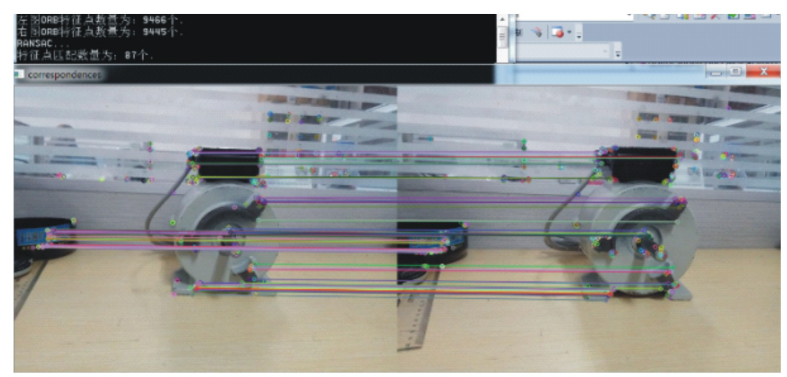

图 4 原始 ORB 特征提取与匹配+RANSAC 外点消除

OGMS, 算法思想基于如下假设：

假设 正确匹配点对周围邻域内的匹配点对应 该具有相同的单位方向向量(见图 5)。

设 $\boldsymbol{M}$ 为某一正确匹配点对邻域内匹配点对连 线单位方向向量构成的矩阵为

$$
\boldsymbol{M}=\left[\begin{array}{lllll}
\boldsymbol{m}_{1} & \boldsymbol{m}_{2} & \boldsymbol{m}_{3} & \cdots & \boldsymbol{m}_{n}
\end{array}\right]^{\mathrm{T}}
$$




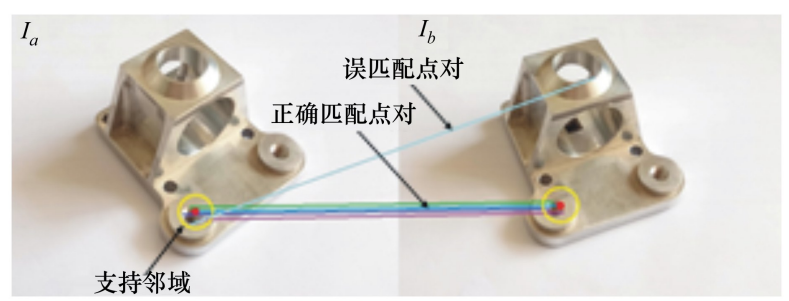

图 5 基于方向向量一致性的特征点匹配假设示意图

方向向量 $\boldsymbol{m}_{i}$ 与 $\boldsymbol{m}_{j}$ 的相似性用点积 $d_{i j}$ 表示

$$
d_{i j}=\left\langle\boldsymbol{m}_{i}, \boldsymbol{m}_{j}\right\rangle
$$

方向向量 $\boldsymbol{m}_{i(1 \times 2)}$ 与匹配点对矩阵 $\boldsymbol{M}_{2 \times n}^{\mathrm{T}}$ 中各方 向向量的相似度用 $G$ 表示

$$
G=\boldsymbol{m}_{i} \boldsymbol{M}^{\mathrm{T}} /(n-1)
$$

将 $G$ 中所有元素按降序排列, 若 $G$ 中某一元素 小于阈值 $\delta$ (实际运算过程取 0.985 ), 说明所对应的 匹配点对的连线方向偏离大部分匹配点对的连线方 向,该匹配点对被作为误匹配而被剔除, 并采用 GMS 中的网格划分进行匹配过程中的加速。

采用上述改进后的 ORB 进行特征匹配结果如 图 6 所示。通过改进的 ORB 对装配环境两相邻视 频帧进行特征提取与匹配, 正确匹配点数目由原来 的 87 变为 3478 ,且该过程耗时较少。

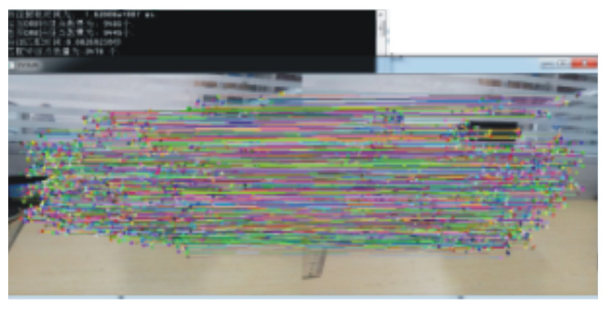

图 6 基于方向向量一致性的 ORB 特征匹配

\section{4 融合视觉特征的 ICP 点云配准}

受 SIFT-ICP 算法的启发 ${ }^{[16]}$, 利用改进的 ORB 特征点匹配, 对 ICP 点云配准, 即传感器位姿估计过 程进行优化。首先从装配环境 2 幅相邻视频帧彩色 图像中分别提取稀疏 ORB 视觉特征集合 $\boldsymbol{F}_{a}, \boldsymbol{F}_{b}$, 通 过改进的 ORB 匹配获得特征点匹配点对集合 $\boldsymbol{C}_{s}$, $\boldsymbol{C}_{t \circ}$ 。因为 ORB 特征是在图像上提取的, 说明在二维 空间特征向量存在相似性, 但是这并不能说明在三 维空间仍然是匹配点对。因此本文首先通过 2 帧图 像对应的深度图像利用 Softkinetic 的内部参数将匹 配点对 $\boldsymbol{C}_{s}, \boldsymbol{C}_{t}$ 映射到三维空间形成空间点云集合 $\boldsymbol{P}_{s}, \boldsymbol{P}_{t}$ 。然后利用随机抽样一致性 (RANSAC) 方法 将三维误匹配点对剔除, 分别形成内点集合 $\boldsymbol{P}$
$\boldsymbol{P}_{t-\text { inliner }}$, 同时获得初始点云变换矩阵 $\boldsymbol{M}^{\prime}=\left[\boldsymbol{R}^{\prime}\right.$ । $\left.\boldsymbol{t}^{\prime}\right]$ 。然后进人 ICP 主循环。在第一次迭代中, 通过 稀疏 ORB 匹配特征点对匹配计算出的初始变换阵 $\boldsymbol{M}^{\prime}=\left[\boldsymbol{R}^{\prime} \mid \boldsymbol{t}^{\prime}\right]$ 使没有任何先验位姿知识的相邻 2 帧 间点云实现了最近邻初始配准。然后将点云配准转 化为求解点云集合间的误差函数最小化问题。误差 函数分为 2 个部分,第一部分计算稀疏 ORB 视觉特 征匹配点对之间的平均欧氏距离,第二部分将当前 点云到目标匹配点云切平面 (point-to-tangent Plane) 的距离作为度量。误差函数用 $(10)$ 式表示

$$
\boldsymbol{M}^{*}=\operatorname{argmin} \alpha
$$

$$
\begin{gathered}
\left(\frac{1}{\left|P_{\text {inliner }}\right|} \sum_{i \in P_{\text {inliner }}} w_{i}\left\|\left(\boldsymbol{R}^{\prime} p_{s_{-} \text {inliner }}^{i}+\boldsymbol{t}^{\prime}\right)-p_{t-\text { inliner }}^{i}\right\|^{2}\right) \\
+(1-\alpha) \\
\left(\frac{1}{\left|P_{d}\right|} \sum_{j \in P_{d}} w_{j}\left\|\left[\left(\boldsymbol{R}^{\prime} p_{a}^{j}+\boldsymbol{t}^{\prime}\right)-p_{b}^{j}\right] \times \boldsymbol{n}_{b}^{j}\right\|^{2}\right)
\end{gathered}
$$

式中, $M^{*}$ 为位姿矩阵, $\alpha$ 为权重值 (实际运算过程 取 0.5$), \boldsymbol{n}_{b}^{j}$ 为稠密点云中目标点 $p_{b}^{j}$ 的法向量, 它是 通过其周边邻域点作叉乘获得。

将装配环境数据点云间满足误差函数最小的匹 配对作为算法的输人量。不同视角图像间的数据可 以看做是多个坐标系间的旋转平移运动, 这样我们 每做一次旋转平移运动就会更新一次数据, 通过反 复迭代变换矩阵, 更新输人数据, 从而最小化环境点 云数据间的偏移误差函数。在点云变换矩阵误差变 化小于阈值 $\delta$ 或者达到最大迭代次数, ICP 主循环 停止迭代,否则将会利用最近一次计算的变换矩阵 进行点云的重新配准, 直到误差满足一定的精度要 求。此过程采用 $\mathrm{kd}$-tree 算法加速最近点搜索, 同时 利用图形处理器 (GPU) 对点云配准过程进行加速 运算。

\section{5 回环检测与位姿全局优化}

连续视频帧间配准方法,适用于短距离的跟踪 注册。当相机移动较长距离时, 相机位姿的估计随 着移动距离的推移而发生漂移, 特别是返回到以前 访问过的位置时, 这种漂移现象最为明显, 这被称为 回环问题。为了解决上述问题, 需要回环检测来识 别相机是否返回到先前访问过的位置。

本文通过判断图像间的相似性来进行回环检 测, 即通过 ORB 视觉特征匹配来完成。首先构建一 个帧间约束的位姿图结构, 它由结点和边组成, 结点 表示每个环境图像帧的位姿,边表示图像帧之间的 
空间位置关系, 它可以通过 RGBD-ICP 点云配准方 法获得。当闭环不存在时, 位姿图结构就是一个线 性链, 当前帧位姿估计误差都会对后续帧产生影响, 相机位姿估计误差较大; 当存在闭环时, 位姿图中就 会产生环形结构, 几个相邻帧间多了一些约束, 因而 误差降低。

为保证位姿图的稀疏性和回环检测的实时性 性, 受文献 [17-18] 启发, 将关键帧作为结点。关键 帧是装配环境图像帧的子集, 其元素个数由装配环 境图像帧的密度和装配环境复杂度来决定。本文关 键帧的选取依据特征点对数来确定。例如, 将第一 帧 $X_{1}$ 作为关键帧, 采用改进的 ORB 提取特征点, 与 其最近邻图像帧进行匹配并获得当前帧与关键帧之 间的变换矩阵。当特征点匹配个数 $N$ 大于阈值 $\phi$ 时, 则不作为下一个关键帧。随着相机的持续移动, 匹配点个数逐渐减少, 将特征点匹配个数 $N$ 小于國 值 $\phi$ 的第一帧作为下一个关键帧。

当新的关键帧 $X_{2}$ 产生后, 与先前每一个关键帧 进行回环检测。为保证检测回环的可靠性, 将匹配 点对利用 Softkinetic 的内部参数和匹配点对计算出 的变换矩阵 $\boldsymbol{M}^{*}=\left[\boldsymbol{R}^{*} \mid \boldsymbol{t}^{*}\right]$ 映射到三维空间, 形成 空间点云集合 $\boldsymbol{P}=\left\{p_{i}\right\}, \boldsymbol{P}^{\prime}=\left\{p_{i}^{\prime}\right\}$ 。当匹配点在三 维空间中匹配误差小于阈值 $\psi$ 时, 可认为闭环产生 了, 并在位姿图结构中添加新的边。匹配误差定 义为

$$
\frac{1}{N} \sqrt{\left\|\sum_{i=1}^{N}\left(\boldsymbol{R}_{\text {relat }} p_{i}+\boldsymbol{t}_{\text {relat }}\right)-p_{i}^{\prime}\right\|^{2}}<\psi
$$

检测到回环后, 根据前后帧计算获得的位姿变 换可以建立前后帧的位姿约束, 回环检测可以建立 回环处的位姿约束, 通过优化这两种约束, 实现前后 帧间的位姿约束与回环约束之间的全局一致性。本 文采用 $\mathrm{TORO}^{[16]}$ 对其进行后台优化, 寻找达到全局 一致性的结点位姿配置, 将累积误差进行均衡处理, 从而优化相机轨迹, 降低相机位姿估计值和真实值 的误差, 提高相机位姿 $\boldsymbol{M}_{\mathrm{RGBD}-\mathrm{ICP}}=\left[\boldsymbol{R}_{\text {relat }} \mid \boldsymbol{t}_{\text {relat }}\right]$ 的计 算精度。

通过相机位姿初始化和 RGBD-ICP 连续视频帧 间配准, 在时刻 $i$ 的相机全局绝对位姿 $M_{i}$ 可用(12) 式表示,其求解过程示意图如图 7 所示。

$$
\boldsymbol{M}_{i}=\boldsymbol{M}_{\text {init }} \boldsymbol{M}_{\mathrm{RGBD}-\mathrm{ICP}}
$$

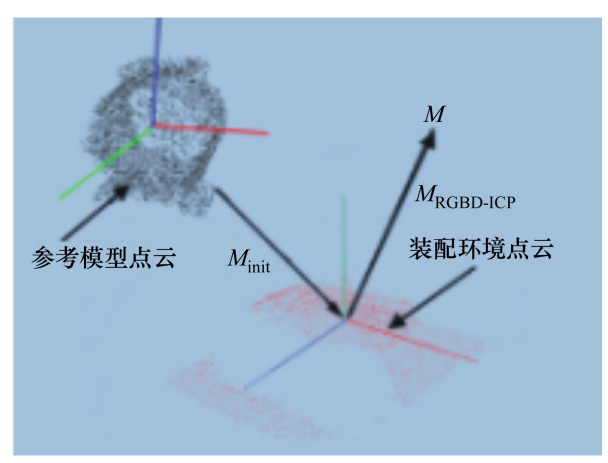

图 7 相机绝对位姿计算示意图

\section{2 方法验证与讨论}

跟踪注册结果可视化实验验证采用英特尔至强 Xeon-E3 微处理器, CPU 主频为 $3.5 \mathrm{GHz}$, 运行内存 为 $8 \mathrm{~GB}$, 显卡为 NVIDIA Geforce GTX 970。 Softkinetic 用于深度和彩色信息获取, 其彩色传感器 分辨率为 $1280 \times 720$, 深度传感器分辨率为 $320 \times$ 240 。为了提高运行速度, 实验中彩色图像大小设 置为 $640 \times 480$ 。软件系统采用 “Unity3D” 游戏引擎 进行开发, 用 3D Studio Max 进行装配引导信息建 模。算法采用 $\mathrm{C}++$ 语言编写, 并以动态链接库 (DLL) 的形式导人 Unity3D。

\section{1 三维跟踪注册算法精确性测试}

在水洜装环境中, 将 Softkinect 深度传感器与虚 拟现实跟踪设备 HTC Vive 手柄 (定位精度 \pm 1.5 $\mathrm{mm}$ ) 绑定, 并将采集到的 HTC Vive 手柄运行轨迹作 为真实值。同时, 分别采用 KinectFusion ${ }^{[19]}$ 跟踪注 册方法和本文提出的点云和视觉特征融合的跟踪注 册方法进行三维注册, 并对 Softkinect 深度传感器运 行轨迹的 $X, Y, Z 3$ 个分量进行分析。实验对比结 果如图 8 所示。从图中可以看出, 传统的基于点云 的跟踪注册方法 (Kinect Fusion) 随着相机移动距离 的增大, 与真实值误差越来越大, 且在 300 帧左右跟 踪注册过程中断, 这是由于相机移动速度过快, ICP 初始对应数据点集获取失败, 导致跟踪注册过程中 断。本文方法在跟踪注册过程中加人了视觉特征匹 配, 因此能获得相机快速移动前后的变换矩阵, 为后 续点云迭代过程提供了正确的初始值, 避免了相机 跟踪注册过程的失败。 


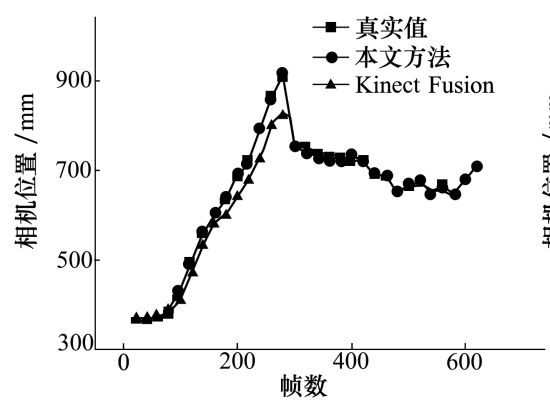

a) $X$ 方向

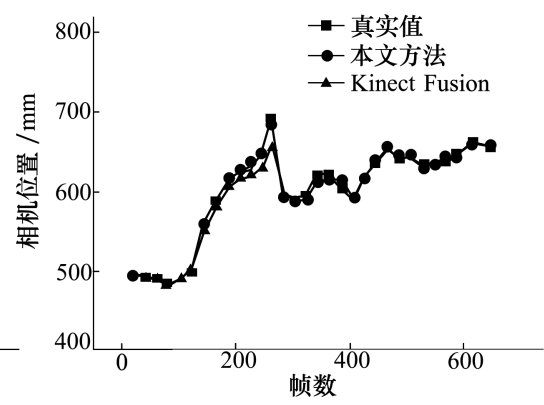

b) $Y$ 方向

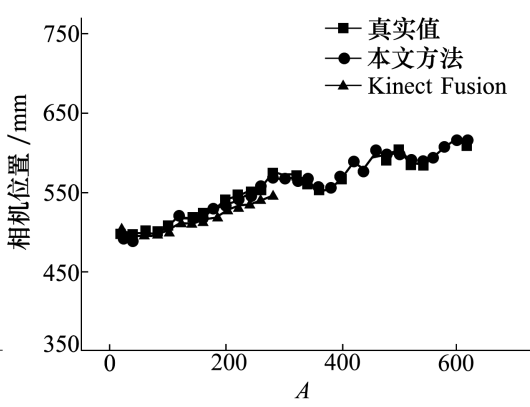

c） $Z$ 方向

图 8 本文方法跟踪轨迹与真实运行轨迹的对比

为了更直观地表达采用不同跟踪注册方法的相 机位置测量值和真实轨迹之间的误差, 采用公式 (13) 进行位置误差的度量:

$$
\begin{gathered}
\varepsilon_{\text {trans }}=\| \text { 测量值 }- \text { 真实值 } \|_{2}= \\
\sqrt{\left(x-x_{\text {真实 }}\right)^{2}+\left(y-y_{\text {真实 }}\right)^{2}+\left(z-z_{\text {真实 }}\right)^{2}}
\end{gathered}
$$

实验结果如表 1 所示。从表中可以看出, 采用 本文方法估计的相机轨迹与真实运行轨迹非常接 近, 相机测量位置与其真实位置误差较小, 表明本文 算法精确性更高。与 KinectFusion 相比, 本文方法 提高了基于稠密点云的跟踪注册准确性和鲁棒性。

表 1 不同跟踪注册方法测量值和 真实值之间的位置误差 $/ \mathbf{m m}$

\begin{tabular}{ccc}
\hline 帧数 & 本文方法 & Kinect Fusion \\
\hline 10 & 6.16 & 10.44 \\
100 & 10.68 & 101.16 \\
200 & 12.21 & 205.35 \\
300 & 12.25 & 311.81 \\
400 & 9.85 & - \\
500 & 9.38 & - \\
600 & 11.36 & - \\
\hline
\end{tabular}

\section{2 跟踪注册算法执行时间分析}

本文跟踪注册过程首先需要利用模型点云完成 相机位姿初始化 (即三维跟踪注册绝对坐标系建 立), 该过程耗时较多 $(\sim 2 \mathrm{~s})$, 但初始化完成后将不 再执行此过程，因此算法执行时间不将其统计在内。 初始化完成后系统跟踪注册过程可分为明显的 4 个 阶段: 深度图像获取、深度图像降噪修复、点云生 成、点云视觉特征融合跟踪注册, 整个过程耗时
$23.19 \mathrm{~ms}$, 系统运行速度可实现每秒 30 帧, 满足系 统实时性要求。

表 2 三维跟踪注册每一帧运行时间

\begin{tabular}{ccc}
\hline 步骤编号 & 执行过程 & 时间 $/ \mathrm{ms}$ \\
\hline 1 & 视频流输人 & 2.86 \\
2 & 点云生成及预处理 & 13.79 \\
3 & RGBD-ICP 跟踪注册 & 4.04 \\
4 & 回环检测 & 2.50 \\
5 & 总时间 & 23.19 \\
\hline
\end{tabular}

\section{3 增强装配}

以水洜作为装配目标, 开发了基于 $\mathrm{AR}$ 的装配 辅助系统。该系统利用 3D Max 进行引导信息建 模, Unity 3D 作为软件开发环境, 本文算法采用 $\mathrm{C}++$ 语言编写, 并以动态链接库 (DLL) 的形式导人 Unity $3 \mathrm{D}$ 。系统硬件配置及软件架构可参阅本团队前期 工作 ${ }^{[7,20]}$ 。将水洜基座三维模型在 CAD 环境中生 成点云模型。同时, 为了呈现良好的虚实融合效果, 采用基于模型的方法 ${ }^{[21]}$ 对装配引导信息和装配体 之间的遮挡关系进行处理。实验过程中, 保持洜体 不动, 手持 Softkinetic 进行移动, 获取不同视角、不 同距离下的装配引导信息三维注册结果, 实验结果 如图 9 所示。图 9a) 为水泵基座, 实验开始后, 首先 获取装配场景深度图像, 生成点云, 并与基座模型点 云进行配准, 获取 Softkinetic 在基座模型坐标系下 的位姿。图 9b) 为初始化完成后, 将基座模型坐标 系原点作为装配基准, 并依据 Softkinetic 位姿, 将洜 体装配引导信息进行叠加。移动 SoftKinetic, 改变其 与基座的视角和距离, 获取不同视角和不同距离下 的引导信息注册结果 (见图 9c) 图 9d)), 从图中 
可以看出, 不同视角、不同距离下本文算法都能达到 较好的三维空间注册效果。图 9e) 图 9g) 展示了 不同装配工序的引导画面, 图中标注所示为动态引 导信息, 用于确定待安装工件洜体和螺栓的装配位
置。对于关键装配工序的装配方法, 采用文字、箭头 指示和动画组合的形式进行引导 (见图 9f)), 便于 操作人员明唽、无二义性地理解装配指令, 提高装配 效率, 降低装配工作出错几率。

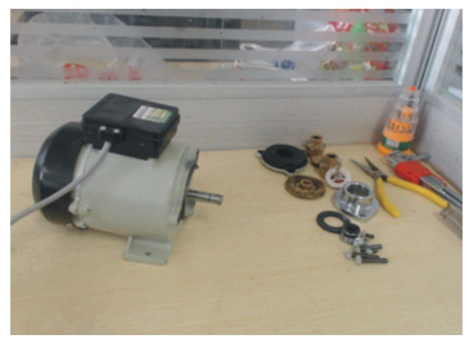

a) 基座

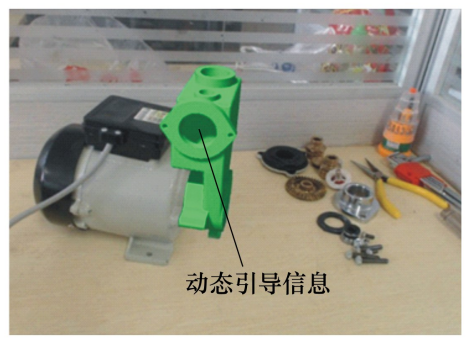

b）原体装配引导信息注册结果

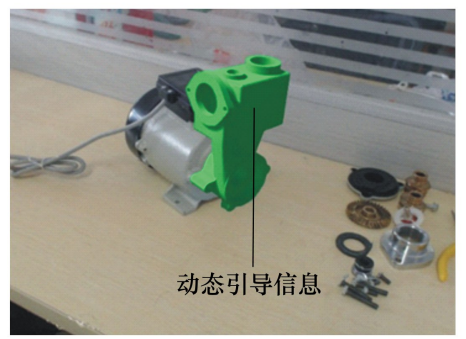

c） 不同视角下原体引导信息注册结果

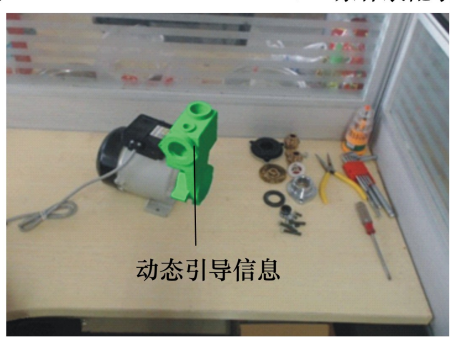

d）不同距离下泵体引导信息注册结果

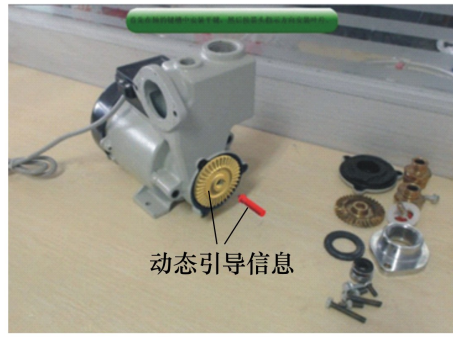

f）叶片装配引导信息注册结果

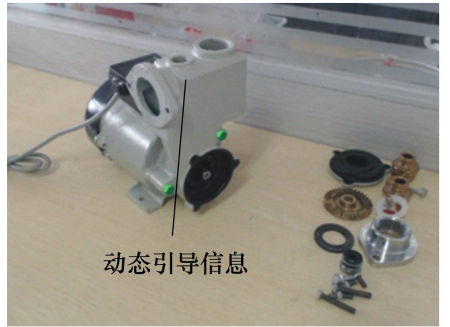

e） 螺旋装配引导信息注册结果

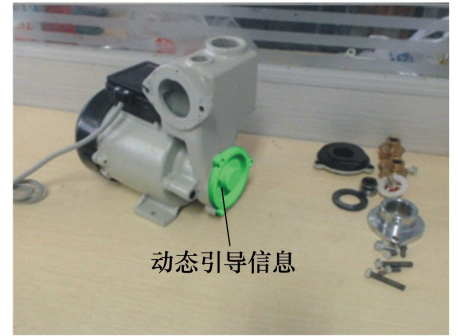

g）端盖装配引导信息注册结果

图 9 三维跟踪注册结果可视化

\section{3 结 论}

本文提出了一种面向 AR 机械装配辅助系统的 三维跟踪注册方法, 该方法首先针对跟踪注册过程 中装配场景物体表面光滑、缺少纹理的特点, 提出了 一种基于方向向量一致性的视觉特征匹配策略。然 后基于 ICP 点云配准方法, 设计了一种点云和视觉 特征匹配相结合的三维跟踪注册方法, 同时加人回
环检测和全局优化策略,既避免了因初始匹配数据 点集不足导致的相机跟踪注册失败, 又保证了相机 位姿估计精度。最后利用三维跟踪注册矩阵, 完成 了基于 AR 辅助系统的水洜装配引导实验。实验结 果表明, 本文方法精确性、实时性好, 能达到每秒 30 帧。而且在相机快速移动时仍能表现出较好的鲁棒 性, 其综合性能优于基于点云的 KinectFusion 三维 跟踪注册方法。

\section{参考文献:}

[1] 刘检华. 军工数字化装配技术 $[J]$. 国防制造技术, 2011(4):5-7

LIU Jianhua. Digital Assembly Technology in Military Industry[J]. Defense Manufacturing Technology, 2011(4) : 5-7 (in Chi- 
nese)

[2] 肖鸿. 面向复杂产品装配现场的移动三维模型关键技术研究 [D]. 西安:西北工业大学,2014

XIAO Hong. Research on the Key Technologies of Mobile Three-Dimensional Model for Assembly Site of Complex Product[D]. $\mathrm{Xi}^{\prime}$ an, Northwestern Polytechnical University, 2014 (in Chinese)

[3] 陈祥辉. 大部件人工装配作业现场信息投影技术研究 $[D]$. 上海:华中科技大学,2016

CHEN Xianghui. Research of Satellite Assembly Induced System Based on Augmented Reality [D]. Shanghai, Huazhong University of Science and Technology, 2016 (in Chinese)

[4] TOMBARI F, FRANCHI A, STEFANO L D. BOLD Features to Detect Textureless Objects [C] //IEEE International Conference on Computer Vision Sydney, 2014: 1265-1272

[5] DAMEN D, BUNnUN P, CALWAY A, et al. Real-Time Learning and Detection of 3D Textureless Objects: a Scalable Approach $[\mathrm{C}] / /$ The British Machine Vision Conference, Guildford, 2012: 1-12

[6] WANG G, WANG B, ZHONG F, et al. Global Optimal Searching for Textureless 3D Object Tracking $[\mathrm{J}]$. Visual Computer, 2015, 31(6/7/8): 979-988

[7] WANG Y, ZHANG S S, YANG S, et al. A LINE-MOD-Based Markerless Tracking Approach for AR Applications [J]. International Journal of Advanced Manufacturing Technology, 2017, 89(5/6/7/8): 1699-1707

[8] 徐迟. 增强现实中的三维物体注册方法及其应用研究 $[D]$. 上海:华中科技大学, 2011

XU Chi. Research on the 3D Objeet Registration Method in Augmented Reality and Its Application[D]. Shanghai, Huazhong University of Science and Technology, 2011 (in Chinese)

[9] ENGEL J, STÜCKLER J, CREMERS D. Large-Scale Direct SLAM with Stereo Cameras [ C ] // IEEE/RSJ International Conference on Intelligent Robots and Systems, Hamburg, 2015: 1935-1942

[10] MUR-ARTAL R, MONTIEL J M M, TARDóS J D. ORB-SLAM: a Versatile and Accurate Monocular SLAM System[J]. IEEE Trans on Robotics, 2015, 31(5): 1147-1163

[11] 付梦印, 吕宪伟, 刘粀, 等. 基于 RGB-D 数据的实时 SLAM 算法 $[\mathrm{J}]$. 机器人, 2015,37(6):683-692

FU Mengyin, LÜ Xianwei, LIU Tong, et al. Real-time SLAM Algorithm Based on RGB-D Data[J]. Robot, 2015, 37(6): 683-692

[12] RADKOWSKI R. Object Tracking with a Range Camera for Augmented Reality Assembly Assistance[J]. Journal of Computing and Information Science in Engineering, 2016, 16(1): 011004

[13] 刘金辰,傅慧源,马华东. 基于 RGB-D 摄像头的实时手指跟踪注册与手势识别 $[\mathrm{J}]$. 计算机科学, 2014,41(10):50-52

LIU Xinchen, FU Huiyuan, MA Huadong, et al. Real-time Fingertip Tracking and Gesture Recognition Using RGB-D Camera $[\mathrm{J}]$. Computer Science, 2014,41(10): 50-52 (in Chinese)

[14] MATSUO T, FUKUSHIMA N, ISHIBASHI Y. Weighted Joint Bilateral Filter with Slope Depth Compensation Filter for Depth Map Refinement [C] //International Conference on Computer Vision Theory and Applications, Berlin, 2015: 313-320

[15] BIAN J W, LIN W Y, MATSUSHITA Y, et al. GMS: Grid-Based Motion Statistics for Fast, Ultra-Robust Feature Correspondence[C] //2017 IEEE Conference on Computer Vision and Pattern Recognition, Puerto Rico, 2017: 2828-2837

[16] 沈跃, 潘成凯, 刘慧, 等. 基于改进 SIFT-ICP 算法的 Kinect 植株点云配准方法 [J]. 农业机械学报, 2017(12)：183-189 SHEN Yue, PAN Chengkai, LIU Hui, et al. Method of Plant Point Cloud Registration Based on Kinect of Improved SIFT-ICP [J]. Transactions of the Chinese Society of Agricultural, 2017(12) : 183-189 (in Chinese)

[17] HENRY P, KRAININ M, HERBST E, et al. RGB-D Mapping: Using Depth Cameras for Dense 3D Modeling of Indoor Environments [M]. Berlin Heidebery, Springer, 2014: 647-663

[18］李同, 张奇志. 基于 ORB 词袋模型的 SLAM 回环检测研究 $[\mathrm{J}]$. 信息通信, 2017(10):20-25

LI Tong, ZHANG Qizhi. Research of SLAM Loop-Closure Based on ORB Bag of Words [J]. Information \& Communications, 2017(10):20-25 (in Chinese)

[19] NEWCOMBE R A, IZADI S, HILLIGES O, et al. Kinect Fusion: Real-Time Dense Surface Mapping and Tracking[C] // IEEE International Symposium on Mixed and Augmented Reality, Basel, 2011:127-136

[20] WANG Y, ZHANG S S, YANG S, et al. Mechanical Assembly Assistance Using Markerless Augmented Reality System[J]. Assembly Automation, 2018, 38(1):77-87

[21] 徐迟,李世其,王峻峰,等. 面向增强现实装配的虚实遮挡技术研究 $[\mathrm{J}]$. 机械设计与制造,2009(12): 256-258 
XU Chi, LI Shiqi, WANG Junfeng, et al. Ocelusion Handling in Augmented Reality Based Virtuecl Assembly [J]. Machinery Oesign \& Manufaeture, 2009(12) : 256-258 (in Chinese)

\title{
A 3D Tracking and Registration Method Based on Point Cloud and Visual Features for Augmented Reality Aided Assembly System
}

\author{
WANG Yue, ZHANG Shusheng, BAI Xiaoliang \\ (School of Mechanical Engineering, Northwestern Polytechnical University, Xi'an 710072, China)
}

\begin{abstract}
To improve the robustness and applicability of 3D tracking and registration for augmented reality (AR) aided mechanical assembly system, a 3D registration and tracking method based on the point cloud and visual features is proposed. Firstly, the reference model point cloud is used to definite absolute tracking coordinate system, thus the locating datum of the virtual assembly guidance information is determined. Then by adding visual features matching to the iterative closest points (ICP) registration process, the robustness of tracking and registration is improved. In order to obtain sufficient number of visual feature matching points in this process, a visual feature matching strategy based on orientation vector consistency is proposed. Finally, the loop closure detection and global pose optimization from key frames are added in the tracking registration process. The experimental result shows that the proposed method has good real-time performance and accuracy, and the running speed can reach 30 frames per second. Moreover, it also shows good robustness when the camera is moving fast and the depth information is inaccurate, and the comprehensive performance of the proposed method is better than the KinectFusion method.
\end{abstract}

Keywords : augmented reality ; mechanical assembly ; 3D registration and tracking; point cloud; visual feature; robustness; visual feature matching; loop closure detection; global pose optimization 